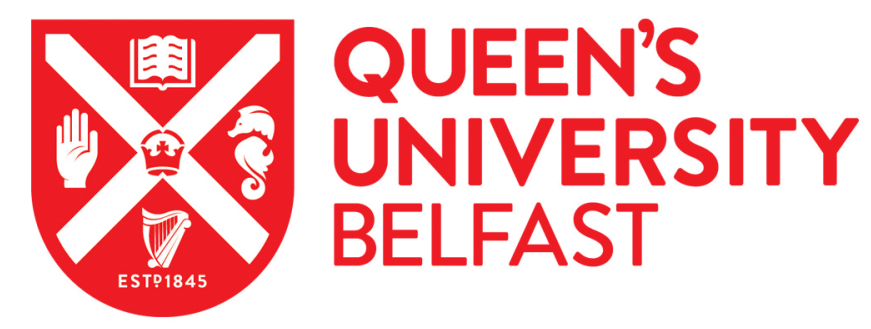

\title{
A Two-tiered system of analysis to tackle rice fraud: The Indian Basmati study
}

Shannon, M., McGrath, T., Elliott, C., \& Ch, R. (2020). A Two-tiered system of analysis to tackle rice fraud: The Indian Basmati study. Talanta, [TAL_122038]. https://doi.org/10.1016/j.talanta.2020.122038

\section{Published in:}

Talanta

\section{Document Version:}

Peer reviewed version

Queen's University Belfast - Research Portal:

Link to publication record in Queen's University Belfast Research Portal

\section{Publisher rights}

Copyright 2020 Elsevier.

This manuscript is distributed under a Creative Commons Attribution-NonCommercial-NoDerivs License

(https://creativecommons.org/licenses/by-nc-nd/4.0/), which permits distribution and reproduction for non-commercial purposes, provided the author and source are cited.

\section{General rights}

Copyright for the publications made accessible via the Queen's University Belfast Research Portal is retained by the author(s) and / or other copyright owners and it is a condition of accessing these publications that users recognise and abide by the legal requirements associated with these rights.

Take down policy

The Research Portal is Queen's institutional repository that provides access to Queen's research output. Every effort has been made to ensure that content in the Research Portal does not infringe any person's rights, or applicable UK laws. If you discover content in the Research Portal that you believe breaches copyright or violates any law, please contact openaccess@qub.ac.uk. 


\section{Journal Pre-proof}

A Two-tiered system of analysis to tackle rice fraud: The Indian Basmati study

Maeve Shannon, Ratnasekhar CH, Terence F. McGrath, Arun P. Kapil, Christopher T. Elliott

PII: $\quad$ S0039-9140(20)31329-1

DOI: $\quad$ https://doi.org/10.1016/j.talanta.2020.122038

Reference: TAL 122038

To appear in: Talanta

Received Date: 28 August 2020

Revised Date: 14 December 2020

Accepted Date: 17 December 2020

Please cite this article as: M. Shannon, R. CH, T.F McGrath, A.P Kapil, C.T Elliott, A Two-tiered system of analysis to tackle rice fraud: The Indian Basmati study, Talanta, https://doi.org/10.1016/ j.talanta.2020.122038.

This is a PDF file of an article that has undergone enhancements after acceptance, such as the addition of a cover page and metadata, and formatting for readability, but it is not yet the definitive version of record. This version will undergo additional copyediting, typesetting and review before it is published in its final form, but we are providing this version to give early visibility of the article. Please note that, during the production process, errors may be discovered which could affect the content, and all legal disclaimers that apply to the journal pertain.

(C) 2020 Published by Elsevier B.V. 


\section{Credit Author Statement}

Maeve Shannon: Conceptualization; Data curation; Formal analysis; Investigation; Methodology Validation; Visualization Roles/Writing - original draft

Ratnasekhar CH: Conceptualization; Data curation; Formal analysis; Investigation; Methodology Validation; Visualization Roles/Writing - original draft

Terry McGrath: Project administration; Supervision; Writing - review \& editing; Supervision

Christopher Elliott: Funding acquisition; Writing - review \& editing; Supervision

Arun Kapil: Sample supplier 
An easy-to-use portable tool to rapidly screen rice at point of

sampling

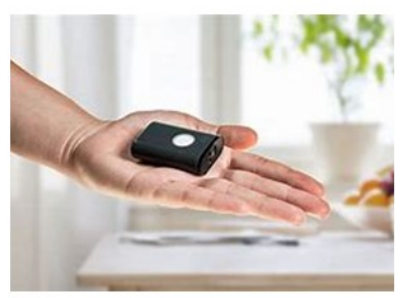

High sample throughput
Suspect rice samples sent for labbased confirmation and quantitation

Agilent GC-MS
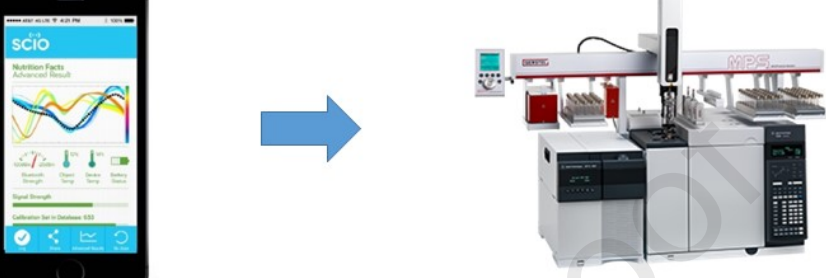
3 Maeve Shannon ${ }^{1 *}$, Ratnasekhar $\mathrm{CH}^{1,2^{*}}$, Terence $\mathrm{F} \mathrm{McGrath}{ }^{1}$, Arun P Kapil ${ }^{3}$ and Christopher T Elliott ${ }^{1}$

$4{ }^{1}$ ASSET Technology Centre, Institute for Global Food Security, Queen's University Belfast

$5 \quad{ }^{2}$ Analytical Chemistry, CSIR-CIMAP, Lucknow, India

$6{ }^{3}$ Green Saffron Spices Ltd, Ireland

$7 \quad$ *Joint primary authors

8

9 Abstract

Demand for high quality Basmati rice has increased significantly in the last decade. This commodity is highly vulnerable to fraud, especially in the post COVID-19 era. A unique two-tiered analytical system comprised of rapid on-site screening of samples using handheld portable Near-infrared NIR and laboratory confirmatory technique using a Head space gas chromatography mass spectrometry (HS-GC-MS) strategy for untargeted analysis was developed. Chemometric models built using NIR data correctly predicted nearly $100 \%$ of Pusa 1121 and Taraori, two high value types of Basmati, from potential adulterants. Furthermore, rice VOC profile fingerprints showed very good classification ( $R 2>0.9, Q 2>0.9$, Accuracy $>0.99$ ) for these high quality Basmati varieties from potential adulterant varieties with aldehydes identified as key VOC marker compounds. Using a twotiered system of a rapid method for on-site screening of many samples alongside a laboratory-based confirmatory method can classify Basmati rice varieties, protecting the supply chain from fraud.

Key words: Basmati rice, Two-tiered method, Adulteration, NIR, Handheld, GC-MS, Chemometrics, Fraud

\section{Introduction}

Rice is a staple food for over half the world's population, and after maize, has the largest cereal production worldwide [1]. Rice can, however, be considered the most important grain for human consumption, as maize is grown for purposes other than human consumption too, such as for animal feed and biofuels [2]. Global consumption of rice has risen in recent years, with about 490 million metric tons of rice being consumed worldwide in 2018/19. Rice has been cultivated in various countries around the world which ultimately increases the diversity of rice in terms of differing varieties and qualities [3]. The range of types of rice available makes the higher quality rice varieties very vulnerable to fraud. 
Food fraud is becoming an increasingly large problem worldwide; the more complicated the food chain becomes, the more opportunity there is for food fraud to occur [4]. As the impacts of the COVID-19 pandemic on the world's food supply system become better known fraud is likely to reach new levels. Despite there being no EU definition of food fraud, it is commonly accepted that "food fraud is an intentional action carried out for financial gain. Different types of food fraud include adulteration, counterfeiting, substitution and deliberate mislabelling of goods" [5]. In recent years, Basmati rice has fallen victim to fraud. The high commercial value of Basmati rice can tempt unscrupulous companies into fraudulent practises and lead them to deceive the consumer [6]. Basmati commands a premium price compared to other long grain rice due to its unique desirable properties [7]. In addition, the demand for Basmati rice has drastically increased over the past decade and another reason why non-Basmati varieties of rice are being mixed with Basmati rice on the world market [6]. In 2014, 22 tonnes of long-grain rice being sold as Basmati were seized in the UK by Interpol and Europol. In this scandal, white long grain rice was being blended with Basmati in order to try and pass it off as Basmati rice [8].

Many screening and confirmatory techniques have been developed to try and identify and combat food fraud. Spectroscopy has been used for many commodities as a fraud screening tool. For example, Fourier Transform Infrared (FT-IR) has been used in a range of herbs and spices such as oregano, sage and black pepper, to detect adulteration [9, 10,11]. Near infrared (NIR) has been used successfully in the detection of Sudan dyes in chili, [12] as well as for milk adulteration [13]. The prospect of developing portable handheld spectroscopy methods is of substantial interest to food distributors and retailers; as well as having some interest to consumers. Using handheld technology allows samples to be tested on-site before going into the marketplace. A 2019 study in Ghana used a handheld NIR and chemometric modelling to classify different qualities of rice and country of origin (COI). Through the use of Matlab software and applying Multiplicative Scatter Correction (MSC) to the data; different quality grades and geographical origin of rice could be classified successfully, with over $90 \%$ of the validation set being correctly classified [14].

In addition to these spectroscopic techniques, a variety of non-targeted techniques, mainly based on mass spectrometry (MS) and nuclear magnetic resonance (NMR), have been used to identify authenticity and food adulteration $[15,16]$. Among these techniques, non-targeted MS is an emerging approach for food authentication studies due to its advantages in terms of sensitivity, rapidity, selectivity and high-throughput analysis $[17,18,19]$. In particular, Headspace solid phasemicro extraction (HS-SPME) directly coupled to gas chromatography-mass spectrometry is one gold standard approach to analyse volatile organic compounds (VOCS). This technique provides a global fingerprint of VOCS for samples, without any sample preparation. HS-SPME-GC-MS has been 
successfully applied to characterize several food matrices (spirits, coffee, honey, juice) for different purposes including food authenticity $[20,21,22,23]$. Basmati rice is well known for its aroma content. The aroma of Basmati rice is a complex mixture of VOCS that is related to several factors including cultivar, origin and quality.

Quite a range of different types of Basmati rice are produced in India and exported worldwide; there are 29 varieties of Basmati notified under the seeds Act, 1966 [24] for Indian export. Some varieties are more expensive than others and as a result, some manufacturers may try to defraud the consumer in order to increase their profits. Therefore, a system which can screen a high volume of samples, i.e. screening by NIR, and a confirmatory identification by HS-SPME-GC-MS combined with multivariate statistical analysis for the VOCS analysis could be implemented in order to tackle substitution fraud issues with Basmati rice.

The aim of the present study was to develop a two-tiered method to identify and protect the more expensive, prized Pusa 1121 and Taraori rice varieties; ensuring that they have not been substituted with lower quality varieties i.e. Pusa 1509, Sugandha, Duplicate Basmati, Shabnam and Sharbati. Pusa 1509 and Sugandha are considered potential adulterants of Pusa 1121. Whilst Shabnam, Duplicate Basmati and Sharbati are considered potential adulterants of Taraori.

\section{Methods and Materials}

\subsection{Sample Collection}

A total of 1399 rice samples were provided by Green Saffron Spices Ltd, Co. Cork, Ireland. The samples comprised of 7 different varieties of Basmati rice. These included two high quality varieties, Pusa $1121(n=250)$ and Taraori $(n=150)$. The 5 other varieties are potential adulterants of either Pusa 1121 or Taraori and included Pusa 1509 ( $n=149)$, Sugandha $(n=250)$, Duplicate Basmati $(n=200)$, Sharbati $(n=150)$ and Shabnam $(n=250)$.

\begin{tabular}{|l|l|l|l|l|}
\hline $\begin{array}{l}\text { Country of } \\
\text { Origin }\end{array}$ & $\begin{array}{l}\text { Name of Basmati } \\
\text { rice variety }\end{array}$ & $\begin{array}{l}\text { Number of } \\
\text { samples } \\
\text { analysed }\end{array}$ & Quality of rice sample & Province \\
\hline \multirow{4}{*}{ India } & Pusa 1121 & 250 & High quality & Punjab \\
\cline { 2 - 5 } & Pusa 1509 & 149 & Adulterant & Haryana \\
\cline { 2 - 5 } & Taraori Basmati & 150 & High quality & Haryana \\
\cline { 2 - 5 } & Duplicate Basmati & 200 & Adulterant & Punjab, Haryana \\
\cline { 2 - 5 } & Sugandha & 250 & Adulterant & Punjab, Haryana \\
\cline { 2 - 5 } & Sharbati & 150 & Adulterant & Punjab, Haryana \\
\cline { 2 - 5 } & Shabnam & 250 & Adulterant & Gujrat \\
\hline
\end{tabular}




\subsubsection{NIR-Instrumentation and SCiO data acquisition}

The SCiO handheld NIR device (Consumer Physics, Israel), was used to acquire spectral data from the rice samples, with a spectral range between $740 \mathrm{~nm}$ and $1070 \mathrm{~nm}$. This works through a phonebased App which records NIR spectra to Consumer Physics' cloud-based database. Users access their data online through a web interface called 'The Lab'.

Before use, the device was calibrated. This was repeated every 10 minutes during analysis of samples. No sample preparation was required for the NIR screening. Each rice sample was dispensed into a glass petri dish $(50 \mathrm{~g})$. The base of the petri dish was then tapped against a hard surface to disperse the sample evenly over the petri dish. Samples were scanned from underneath and the petri dish was held static in location during each scan and only moved after a scan was completed and before a new scan began. The petri dish was rotated between each scan to ensure different areas of the sample were scanned for each replicate. Samples were scanned in triplicate. Spectral data was stored in the cloud and was downloaded from 'The Lab' and imported to SIMCA 15 (Sartorious, Sweden) for chemometric model building. Models were also built using 'The Lab' software.

\subsubsection{NIR-Chemometric modelling}

The raw spectral data (Figure $1 \mathrm{~A}$ ) had a number of algorithms applied prior to model building in SIMCA 15. The pre-processing methods used on the spectral data were Standard Normal Variate (SNV), First derivative and Savitzky-Golay (SG). Pre-processing of the spectral data is a fundamental part of chemometric modelling in terms of eliminating background noise from the spectra.

Both multiclass and binary models were built using supervised techniques including partial least square discriminant analysis (PLS-DA) and orthogonal partial least square discriminant analysis (OPLS-DA). Models built included i) all varieties of rice, ii) 1121 vs its adulterants (multiclass), iii) 1121 vs its adulterants (binary), iv) Taraori vs its adulterants (multiclass), v) Taraori vs its adulterants (binary). The Q2 gives an estimate of the theoretical predictive ability of the model, however using an external Validation set provides a more robust assessment. This was achieved by using two thirds of the samples as the Reference set and one third as the Validation set. Practically, samples were grouped by variety and each group was in turn randomly split into Reference set and Validation set using the "RAND" function in Microsoft Excel in order to eliminate selection bias. For the models 
containing all varieties, the Reference set included 934 samples, with the Validation set including 465 samples.

'The Lab' software enables the creation of Random Forest (RF) models, a supervised learning algorithm, in order to classify different groups or varieties of samples. Basically, RF builds numerous decision trees and combines them together to get a more accurate and stable prediction. However, it is important to note that RF models do differ from decision trees, for example, decision trees may be vulnerable to overfitting, whereas RF models can overcome this by creating random subsets of the features and building smaller trees using those subsets. The subsets are then merged together. Before building the RF models it is possible to pre-process the data in various ways. Supplementary data: Table 1 summarises the different combinations of pre-processing techniques used in this study and abbreviations (if applicable).

One class classifier model, Data-Driven Soft Independent Modelling of Class Analogy (DD-SIMCA) [25] was performed using MATLAB R2019B, DD-SIMCA tool box [26]. This one class classifier method enabled to distinguish objects of one particular target class, from all other objects and classes. This model is able to compute the misclassification errors theoretically.

\subsection{GC-MS}

\subsubsection{Sample preparation and SPME}

Three grammes of the rice samples were precisely weighed into a $20 \mathrm{~mL}$ glass headspace vial. The headspace in the vial was equilibrated at $70^{\circ} \mathrm{C}$ for $6 \mathrm{~min}$ and then volatile compounds were extracted using DVB/CAR/PDMS fibers (SUPELCO, Bellefonte, PA, USA) at $70^{\circ} \mathrm{C}$ for $5 \mathrm{~min}$. Before applying to the rice samples, the fiber was previously conditioned at $270^{\circ} \mathrm{C}$ for $30 \mathrm{~min}$ in accordance with the manufacturer's instructions. The volatile aroma compounds were desorbed in the GC inlet at $250^{\circ} \mathrm{C}$ for $2 \mathrm{~min}$ in spitless mode and the fiber was conditioned for $5 \mathrm{~min}$ at $270^{\circ} \mathrm{C}$ prior to each analysis.

\subsubsection{GC-MS analysis}

GC-MS analysis was carried out using an Agilent 7890A instrument (Agilent Technologies, Inc., Santa Clara, CA, USA) equipped with an Agilent 7010 Mass selective detector. DB-5MS column (30 m x 0.25 $\mathrm{mm} \times 0.25 \mu \mathrm{m}$ ) (Agilent Technologies, Inc., Santa Clara, CA, USA) was used for chromatographic separation of volatile compounds. The column oven programme was maintained initially at $50^{\circ} \mathrm{C}$ for $1 \mathrm{~min}$, and then heated to $170^{\circ} \mathrm{C}$ at $10^{\circ} \mathrm{C}$ per min, held for $2 \mathrm{~min}$, and heated at $30^{\circ} \mathrm{C}$ per min to a 
final temperature of $280^{\circ} \mathrm{C}$, which was maintained for $1 \mathrm{~min}$. The temperature of the gas chromatograph inlet was maintained at $250^{\circ} \mathrm{C}$ and samples were analyzed in spitless mode. Helium was used as a carrier gas at a flow rate of $1 \mathrm{~mL} / \mathrm{min}$. The mass spectrometer was operated in electron impact ionization mode with ionization energy of $70 \mathrm{eV}$, and the temperature of the ion source was $230^{\circ} \mathrm{C}$. The temperature of the quadrupole and transfer line maintained at $150^{\circ} \mathrm{C}$ and $280^{\circ} \mathrm{C}$, respectively. Analyte signals were collected in full scan mode from 30 to 550 at $5.2 \mathrm{scans} / \mathrm{sec}$. The identification of volatile compounds was performed with NIST14 library and the confirmation of identification was performed by comparing the compounds spectra with literature reported values.

\subsubsection{Statistical analysis}

Total area normalization for each sample was performed in the data matrix (Rt, $\mathrm{m} / \mathrm{z}$ and samples). Pareto Scaled data (mean centred and divided by VSD) was used for multivariate analysis to remove the offsets and adjust the importance of high and low abundance analytes to an equal level. In the present study for the analysis of rice sample datasets, initially unsupervised Principal component analysis (PCA) was performed. PCA summarizes information from the original data having multivariate datasets and reveals the trends in datasets. PCA transforms large number of original correlated variables into several independent uncorrelated variables, which have the ability to account for most of the variation in the original variables (27). Furthermore, a supervised pattern recognition technique, PLS-DA, was used in order to classify samples based on quality. PCA and PLSDA models were built evaluating the proper number of principal components and latent variables, respectively. The developed PLS-DA model was validated using the 'leave one out cross' validation method and its quality was assessed based on $R^{2}$ and $Q^{2}$ scores [28]. Furthermore, this model was validated using 100 times permutation tests [28, 29, 30]. PLS-DA model generates Variable Importance in Projection (VIP) scores. Volatile organic compounds with VIP values greater than 1 were identified as potential differential compounds. Univariate statistical analysis, one-way ANOVA followed by post hoc Fisher's LSD test $(p<0.05)$ with FDR cut-off 0.05 , was performed to assess the difference between mean peak ratios of identified volatile molecules of rice samples of different quality. Multivariate statistical analysis was performed by using the online platform, Metaboanalyst (www.metaboanalyst.ca) [31].

\subsubsection{Tentative identification of VOCs}

Automated Mass Spectral Deconvolution and Identification System (AMDIS) was performed for deconvolution, and the deconvoluted spectra were compared with spectra available from NIST library (a match quality of 90\% minimum was used as a criteria ( $v 2.2 \mathrm{~g}$ distributed with NIST 2014, USA) [32] or Golm metabolome database [33]. Agilent compound discovery software was used to 
identify volatile organic compounds present in rice samples. In addition, previous literature was used to match compound spectra.

\section{Results and discussion}

Varieties of Basmati such as Pusa 1121 and Taraori are highly prized in the international market with regards to their unique grain and cooking/eating quality. Taraori is a traditional Basmati variety, whereas Pusa 1121 is an evolved Basmati variety from Karnal, Haryana market. Research has indicated that due to the higher yield and unique grain and cooking quality traits of Pusa 1121, there is high acceptance on the domestic and global market, which is turn encourages millers to pay a premium price to the farmers [34]. In order to identify and help protect Pusa 1121 and Taraori rice varieties, ensuring that they have not been substituted with the less expensive and in some cases, lower quality rice, a two-tiered method has been developed and validated in this study.

\subsection{NIR screening for the analysis of Indian Basmati rice samples}

For the first tier analysis, all samples were scanned using the SCiO instrument and chemometric models were built using SIMCA 15 and 'The Lab' software to determine if the varieties could be differentiated. An independent data set was then used to test all multiclass and binary (2-class) models.

\subsubsection{Multiclass models}

In order to determine whether all varieties could be differentiated, models containing all 7 varieties (Reference set $n=934$ ) were produced and validated (Validation set $n=465$ ). The raw NIR spectra of all samples and the corresponding multiclass models is shown in Figure 1. Clustering within varieties and in many instances, variety separation can be observed. However, there is some overlap between Sugandha, Pusa 1509 and Duplicate Basmati. Ultimately this is not an issue as the higher quality Pusa 1121 and Taraori varieties, remain separate from the others. The best performing multiclass models developed in SIMCA 15 are summarised in Table 1 and expanded on in Supplementary data: Table S2. Both $M 1$ and $M 3$ performed similarly in terms of correctly identifying rice varieties. Other models performed poorly when correctly identifying individual varieties in the prediction, for example, M2 and M4 which have low performance for Pusa 1509 (Supplementary data: Table S2). In this case, Pusa 1509 was misclassified as either Sugandha or Duplicate Basmati. This can be expected when there are so many different varieties in one model. Therefore, it is more beneficial to focus the models to address "real life" scenarios i.e. create models containing the variety that needs 
221

222

223

224

225

226

227

228

229

230

231

232

233

234

235

236

237

238

239

240

241

242

243

244

245

246

247

248

249

250

251

252

253

protected with its potential adulterants, as opposed to having a large model containing all the varieties.

Therefore, multiclass models containing Pusa 1121 and its potential adulterants, Sugandha and 1509 only, were built (Reference set $n=434$ ). From the performance characteristics, all models show $100 \%$ correct classification of 1121 in the validation set, (Validation set $n=215$ ). There was some misclassification with the adulterants, however, none of which were classified as 1121; they were misclassified as either 1509 or Sugandha. This finding is evident in the model (M10), where there is some overlap between Sugandha and 1509 (data not shown) and its performance characteristics are presented in Table 1. Similarly, multiclass models containing Taraori and its three potential adulterants; Sharbati, Duplicate Basmati and Shabnam were generated in SIMCA 15 (Reference set $n=500$ ) and validated (Validation set $n=250$ ). There was good separation of these four varieties (data not shown). M13 and M15 performed equally well with 100\% correctly identified Taraori and the highest rates of correctly predicted Duplicate Basmati, Sharbati and Shabnam (Table 1). No Shabnam samples were misclassified. Only one sample of Duplicate Basmati was misclassified as Shabnam and one Sharbati samples was below the cut off value. Therefore, importantly, none of the potential adulterants were misclassified as Taraori.

A number of Random Forest (RF) multiclass models were built in 'The Lab' software using the same model set and validation set as was used to build the SIMCA 15 models. Twenty-nine different combinations of pre-processing techniques were applied to the spectra for each type of model. The first type of models generated were those containing all 7 varieties. Out of the 29 models built for this type, the best performing model was selected based on the internal validation and also, more importantly, the evaluation of the external validation set. The best performing model was obtained after averaging the sample replicates then applying, in order: SNV -Avg Dtrend (RF18), to the full spectral range produced by the SCiO (Table 1) (Supplementary data: Figure S1A). None of the adulterants in the Validation set were misclassified as Pusa 1121 or Taraori, but were misclassified as another potential adulterant in some instances. Pusa 1121 was not misclassified as any other variety, but one sample was returned as "Undefined". This was also the case for the Taraori.

The next type of multiclass models built using 'The Lab' contained Pusa 1121 and its adulterants, Sugandha and 1509 only. Again, 29 different models were built and the best performing was selected through external validation in the interest of robustness (Table 1). The best performing models were obtained after averaging the sample replicates then applying, in order: Log; SNV; Subtract Average; Second Derivative (window: 35, quadratic polynomial); and Wavelength Z-score (RF52), as well as Log; SNV; Subtract Minimum; Second Derivative (window: 35, quadratic 
polynomial); and Wavelength Z-score (RF54) to the full spectral range produced by the SCiO (Supp data: Figure S1B). In the cases of both these models, no Pusa 1121 samples were misclassified as the potential adulterants and neither Pusa 1509 or Sugandha were misclassified as Pusa 1121.

The final multiclass models built using the 'The Lab' contained Taraori and its adulterants. The 29 different models were built using different combinations of pre-processing techniques. A model which, following averaging and then applying, in order: Log; SNV and Wavelength Z Score (RF79), resulted in no misclassification of adulterants as Taraori; any samples not classified correctly, were "Undefined". Similarly, only 1 of Taraori samples were misclassified and again this resulted in an "Undefined" classification (Supp data: Figure S1C) Therefore, in this model, no Taraori samples were classified as any of the potential adulterants, nor were any of the potential adulterants classified as Taraori. The performance characteristics for this model are summarized in Table 1.

\subsubsection{Binary (2-Class) models}

Binary models were also generated in SIMCA 15 and 'The Lab' software. In these 2 class models, the samples are classed as either "Pusa 1121" or "Adulterant" or "Taraori" or "Adulterant".

Various 2 class models containing "Pusa 1121" and "Adulterant" were developed and validated using the SIMCA 15 software. The models were tested with an independent data set. Figure 2a shows an example of the binary model, developed in SIMCA 15, for "Pusa 1121" vs "Adulterant". When models M9 and M10 were tested with an independent data set, there was no misclassification of the samples (Table 2). The same was also done for "Taraori" vs "Adulterant" and an exemplar model is shown in Figure $2 \mathrm{~b}$. Even though visually there appears to be an overlap between the two classes in Figure $2 b$, when models M13 and M15 were tested with the independent data set, there was no misclassification of the samples (Table 2).

Similarly, binary RF models were built in 'The Lab' software using different combinations of preprocessing techniques. The first type of binary models was created to distinguish between "Pusa 1121" and "Adulterant" (Supplementary data: Figure S2A). When an independent data set was tested against the models, the performance of all models was quite high, with Pusa 1121 correctly predicted $100 \%$ and adulterant correctly $98.5 \%$. A number of models performed equally well (RF109 and RF110) following testing with the Validation set (Table 2). When these models were tested, $100 \%$ of Pusa 1121 samples were identified correctly and only $1.5 \%$ of adulterants were misclassified as Pusa 1121.

The final type of binary models produced by 'The Lab' were those distinguishing between two classes; "Taraori" and "Adulterant". Models obtained after averaging sample replicates, then 
applying, in order: Log; SNV; Subtract Average; First Derivative (window: 35, quadratic polynomial) and Wavelength Z-score, as well as Log; SNV; Subtract Average; Second Derivative (window: 35, quadratic polynomial) (Supplementary data: Figure S2B), performed the highest when the independent data set was tested against the models. The performance of these models summarized in Table 2.

\subsubsection{Chemometric analysis using DD-SIMCA}

DD-SIMCA, an authentication technique is widely used for quality assessment of samples based on NIR analysis. This method consists of two-steps. The first step involved applying PCA to the training data set from the target class. After deriving principal components, in the second step, the training set of authentic samples is used for classification and it is possible to calculate a score distance (SD), the orthogonal distance (OD). SD and OD characterizes the distance of the sample to the score space and it is possible to develop an acceptance area decision rule for a given type 1 error $(\alpha)$.

In the models, high quality rice samples are considered as a training set and tested with the new set from adulterants. In the first case, 167 samples from 1121 was considered as training set and tested with adulterants consisting of 132 samples from both 1509, Sugandha. In order to obtain optimum classification with DD-SIMCA five principal components were considered. All samples inside the area (green objects) were considered as members of target class and samples outside the area considered as extreme (orange objects). Two samples of 1121 of training set fell outside of the acceptance area were classified as extreme. There are no outliers found in the acceptance plot of training set. Three samples of testing set from 1509 and Sugandha fell outside of the test set samples. The models of the acceptance plots for a given $(\alpha)$ values for training and test sets are shown in Figure 3A. 97.7\% sensitivity and 98.8\% specificity were obtained for 1121 vs 1509, Sugandha rice samples. Similarly, in the second case, 100 samples from Taraori was considered as training set and tested with adulterants consisting of 200 samples from Duplicate basmati, Shabnam and Sharbati samples. One extreme and no outliers found from training set of Taraori. Four objects fell outside from testing set of Duplicate basmati, Shabnam and Sharbati samples. The models of the acceptance plots were shown in Figure 3B. 99\% sensitivity and 98\% specificity were obtained for this model. The summary of DD-SIMCA performance was shown in figure 3C.

In the third case, high quality samples, 1121 and Taraori rice samples are tested individually with each adulterant. Samples from 1121 were tested individually with training set of 49 samples from 1509 rice samples and 89 samples from Sugandha rice samples (figure 4A). Similarly, Taraori samples were tested individually with 67 samples of Duplicate basmati, 78 samples of Shabnam and 50 samples Sharbati samples (figure 4B). In all cases more than $98 \%$ of specificity and $95 \%$ of specificity 
were obtained. The models of the acceptance plots for training and test sets and the summary of DD-SIMCA performance are shown in Figure 4.

\subsection{HS-SPME-GC-MS analysis of VOCS for Indian Basmati rice samples}

To identify the qualitative differences between the rice sample groups, as a second tier, untargeted VOCS metabolomics profiling was performed using SPME-GC-MS analysis. Marker compounds were revealed with the aid of multivariate pattern recognition analysis.

Total ion chromatogram of Pusa 1121, Sugandha, Pusa 1509 rice VOCS profiles are shown in Figure S3A. Unsupervised PCA modelling was performed to reduce the data to low dimensional space, where discrimination of VOCS profiles between sample classes can be modelled. The PCA model obtained from Pusa 1121, Sugandha, Pusa 1509 rice samples revealed the general structure of the complete data set, in which the first principal component (PC1) explained $53.6 \%$ and the second principal component explained $22 \%$ of the total variance respectively (Figure $5 \mathrm{~A}$ ). Clear clustering was observed between Pusa 1121, Sugandha and Pusa 1509. PC2 explained the variation of high quality Pusa 1121 from the adulterants, Sugandha and Pusa 1509. PLS-DA and internal cross validation was further performed to find out the predictive accuracy and fit of the polynomial model. The cumulative values of PLS-DA model with an accuracy of $1.0, R^{2}=0.99813$ and $Q^{2}=0.97874$ shows good fit of the model (Supp data: Figure S3B). To assess the statistical significance of these apparently highly predictive multivariate models, permutation testing was conducted. The supervised models were further validated with 100 times permutation tests (Supp data: Figure S3C). From the analysis of these distributions, the significance of the power of the optimal models to predict the VOCS profiles of Pusa 1121, Sugandha and Pusa 1509 was determined to be $p<0.01$. Among all the different variables selected according to the VIP values from the PLS-DA model (VIP $>1$ ), 9 organic compounds, include Hexanal, Nonanal, Heptanal, Heptanoic acid, Nonanoic acid, 2-Acetyl pyrrolidine, 1-Hexanol, 3-Nonen-1-ol and Hexane,2,3,4-trimethyl (Figure 5B; Supplementary data: Table S3). Aldehydes are secondary metabolites and contribute to the flavour notes to rice. In the present study, the aldehydes, Hexanal, Nonanal, and Heptanal are highly enriched in high quality Pusa 1121 samples. In addition to these, 2-acetyl-pyrrolidine, a well-known marker for rice aroma is found to be high in Pusa 1121 samples in comparison to Sugandha and Pusa 1509 samples. Alcohols, 1-Hexanol is relatively high in Pusa 1121 samples in comparison to Sugandha and Pusa 1509, while 3Nonen-1-ol is found to be relatively low level in Sugandha in comparison to Pusa 1121. Organic acids, heptanoic acid, nonanoic acid were found to be relatively high levels in adulterant sample groups, Sugandha and Pusa 1509. Overall, marker compounds, hexanal, nonanal, heptanal and 2-acetyl- 
pyrrolidine, 1-hexanol and hexane-2,3,4-trimethyl, can be used to identify high premium quality Pusa 1121 from other two varieties (Pusa 1509 and Sugandha), while 3-Nonene-1-ol, heptanoic acid, nonanoic acid can be used to distinguish Sugandha rice samples from the other two varieties (Pusa 1121 and Pusa 1509).

Total ion chromatograms of Taraori, Sharbati, Duplicate Basmati and Shabnam rice VOCS profiles are shown in Supplementary data (Figure S4A). The data of these samples were interrogated using unsupervised PCA modelling which established a classification between high quality Taraori and adulterants, Sharbati, Duplicate Basmati and Shabnam rice samples (Figure 5C). The cumulative values of PLS-DA model with accuracy of $1.0, R^{2}=0.99887, Q^{2}=0.99245$ shows good fit of the model (Supplementary data: Figure S4B). The models were further validated with 100 times permutation tests (Supplementary data: Figure S4C). From the analysis of these distributions, the power of the optimal models to predict the VOCS profiles of Taraori and Sharbati, Duplicate Basmati and Shabnam was found to be significant $(p<0.01)$. A total of 9 differential VOCS compounds were identified to differentiate high quality Taraori sample group from adulterant groups Sharbati, Duplicate Basmati and Shabnam rice (Figure 5D) and the list of identifying differential metabolites with VIP scores, along with $p$ values are shown in Supplementary data: Table S4. These compounds include hexanoic acid, pentanoic acid, nonanoic acid, hexanal, heptanal, propanal-2-methyl, benzaldehyde-4-propyl, alpha-ylangene, 1-hexanol-2-ethyl, 2-propyl-1-pentanol. Organic acids, pentanoic acid, nonanoic acid were found to be less in high quality Taraori samples, while hexanoic acid is found to be high levels in duplicate and Shabnam rice samples. Aldehydes, hexanal, heptanal, Propanal-2-methyl, Benzaldehyde,4-propyl were found to be relatively highly enriched in high quality Taraori samples in comparison to Sharbati, Duplicate Basmati and Shabnam rice samples. In addition to these, alcohols, 1-hexanol-2-ethyl, 2-propyl-1-pentanol and terpene, alpha-ylangene were found to be relatively high level in high quality Taraori rice samples in comparison to adulterant rice samples, Sharbati, Duplicate Basmati and Shabnam rice samples. Overall, VOCs, hexanal, heptanal, propanal-2-methyl, benzaldehyde-4-propyl, alpha-ylangene, -1-hexanol-2-ethyl, and nonanoic acid can be used to distinguish high premium quality Taraori rice samples from other three varieties (Duplicate Basmati, Shabnam and Sharbati), while hexanoic acid and pentanoic acid can be used to distinguish Duplicate and Shabnam rice samples from other two varieties (Sharbati and Taraori).

Overall, the high-quality rice samples Pusa 1121 and Taraori contains relatively high levels of aldehydes, alcohols. In contrast, low-quality rice samples, Sugandha, Pusa 1509, Sharbati, Duplicate Basmati and Shabnam samples contain relatively high levels of organic acids.

\section{Conclusion}


The study has shown that handheld NIR and HS-SPME-GC-MS can be used for the successful screening and confirmation of adulterated Indian Basmati rice samples. The major advantage of a two-tiered system is that a large number of samples can be screened in-field using the handheld NIR and non-conforming samples can be sent for confirmatory analysis; cutting down on both time and cost. A two-tiered approach was carried out previously for herb authenticity testing. In that study, a combination of benchtop FT-IR and liquid-chromatography high resolution mass spectrometry (LCHRMS) was used to screen for and confirm oregano adulteration (Black et al., 2016). Although the instruments used differ from the present study, the approach of coupling a screening method with a confirmatory method remain the same. The two software applications used for NIR samples screening produced similar performing models, however the advantage of using 'The Lab' software to produce and validate models is that a sample can be scanned on site and an immediate result given via the App.

Therefore, the handheld SCiO device facilitates rapid results, aiding rapid decision making across supply chains and is easy to use. However, it must be remembered that it is only a screening tool, which is where the confirmatory method comes in to use. If a non-compliant sample is found through the screening method, it can then be sent for confirmatory analysis based on the GC-MS profiling of VOC maker compounds. This study clearly indicates that using a two-tiered system of a rapid screening method alongside a confirmatory method is appropriate to classify Indian Basmati rice varieties and thus help further protect the integrity of the Basmati rice supply chain.

\section{Acknowledgements}

The authors would like to thank the Agilent Foundation and Mars Global Food Safety Center China for their support throughout this project.

\section{References}

1. Lantin, R. (1991) FAO. Rice: Post-harvest Operations. Accessed: 01.05.2020. Available from: http://www.fao.org/3/a-ax442e.pdf.

2. OECD/Food and Agriculture Organisation of the United Nations (2015) OECD-FAO Agricultural Outlook 2015, OECD Publishing, Paris. The Setting: Divergence of crop and livestock markets in 2014, p.29.

3. Shahbandeh, M. (2019) Total rice consumption worldwide from $2008 / 2009$ to $2018 / 2019$. Accessed: 13.02.2020. Available from: https://www.statista.com/statistics/255977/totalglobal-rice-consumption/. 
4. McGrath, T.F., Haughey, S.A., Patterson, J., Fauhl-Hassek, C., Donarski, J., Alewijn, M., van Ruth, S., and Elliott, C.T. (2018) What are the scientific challenges in moving from targeted to non-targeted methods for food fraud testing and how can they be addressed? Spectroscopy case study. Trends in Food Science \& Technology, 76, 38-55.

5. EU. (2014). European Parliamentary Research Service (EPRS) briefing $(16 / 01 / 2014)$ on fighting food fraud. Available from: http://www.europarl.europa.eu/RegData/bibliotheque/briefing/2014/130679/LDM_BRI(201 4)130679_REV1_EN.pdf.

6. Fridez, Françoise (2016) Basmati rice fraud under the magnifying glass of DNA analysis. CHIMIA International Journal for Chemistry, 70 (5) 354-356.

7. Bhattacharjee, P., Singhal, R.S., and Kulkarni P.R. (2002) Basmati rice: a review. International Journal of Food Science and Technology, 37, 1-12.

8. Glotz, J (2014) The Grocer. Fake vodka and Basmati rice seized in UK in food fraud crackdown. Available from: https://www.thegrocer.co.uk/food-safety/fake-vodka-andbasmati-rice-seized-in-uk-in-food-fraud-crackdown/354618.article

9. Black, C., Haughey, S., Chevallier, O.P., Galvin-King, P., and Elliott, C. (2016) A comprehensive strategy to detect the fraudulent adulteration of herbs: The Oregano approach. Food Chemistry, 210, 551-557.

10. Galvin-King P, Haughey SA, Montgomery H, Elliott CT. (2019) The Rapid Detection of Sage Adulteration Using Fourier Transform Infra-Red (FTIR) Spectroscopy and Chemometrics. 102(2):354-362.

11. Wilde, A.S., Haughey, S.A., Galvin-King, P., and Elliott, C. (2019) The feasibility of applying NIR and FT-IR fingerprinting to detect adulteration in black pepper. Food Control, 100, 1-7.

12. Haughey, S.A., Galvin-King, P., Ho, Y.C., Bell, S.E.J. and Elliott, C. (2015) The feasibility of using near infrared and Raman spectroscopic techniques to detect fraudulent adulteration of chili powders with Sudan dye. Food Control, 48, 75-83.

13. Kasemsumran S, Thanapase W, Kiatsoonthon A. (2007) Feasibility of near-infrared spectroscopy to detect and to quantify adulterants in cow milk. Analytical Science. 23(7):907-10.

14. Teye, E., Amuah, C.L.Y., McGrath, T., and Elliott, C. (2019) Innovative and rapid analysis for rice authenticity using hand-held NIR spectrometry and chemometrics. Spectrochimica Acta Part A: Molecular and Biomolecular Spectroscopy, 217, 147-154. 
15. Shi T, Zhu M, Chen Y, Yan X, Chen Q, Wu X, Lin J, Xie $1 \mathrm{H}$. NMR combined with chemometrics for the rapid detection of adulteration in camellia oils. (2017) Food Chemistry. 242, 308-315.

16. Hatzakis, E. (2019), Nuclear Magnetic Resonance (NMR) Spectroscopy in Food Science: A Comprehensive Review. Comprehensive Reviews in Food Science and Food Safety, 18: 189220.

17. Black, C., Chevallier, O.P., and Elliott, C. (2016) The current and potential applications of Ambient Mass Spectrometry in detecting food fraud. TrAC Trends in Analytical Chemistry, $82,268-278$.

18. Ellis, D.I., Muhamadali, H., Allen, D.P., Elliott, C., and Goodacre, R. (2016) A flavour of omics approaches for the detection of food fraud. Current Opinion in Food Science, 10, 7-15.

19. Gerbig, S., Neese, S., Penner, A., Spengler, B., and Schulz S. (2017) Real time Food Authentication using a miniature mass spectrometer. Analytical Chemistry, 89 (20) 1071710725.

20. Peng, J., Yang, Y., Zhou, Y., Hocart, C.H., Zhao, H., Hu, Y., and Zhang, F. (2020) Headspace solid-phase microextraction coupled to gas chromatography mass spectrometry with selected ion monitoring for the determination of four food flavoring compounds and its application in identifying artificially scented rice. Food Chemistry, 313, 126136.

21. Jeleń, H. H., Ziolkowska, A., and Kaczmarek, A. (2010). Identification of the botanical origin of raw spirits produced from rye, potato, and corn based on volatile compounds analysis using a SPME-MS method. Journal of Agricultural and Food Chemistry, 58, 12585-12591.

22. Ravid, U., Elkabetz, M., Zamir, C., Cohen, K., Larkov, O. and Aly, R. (2010) Authenticity assessment of natural fruit flavour compounds in foods and beverages by auto-HS-SPME stereoselective GC-MS. Flavour and Fragrance Journal, 25, 20-27.

23. Yang, Y.Q., Yin, H.X., Yuan, H.B., Jiang, Y.W., Dong, C.W., and Deng, Y.L. (2018) Characterization of the volatile components in green tea by IRAE-HS-SPME/GC-MS combined with multivariate analysis. PLoS One, 13(3) e0193393.

24. APEDA (Agricultural \& Processed Food Product Export Development Authority). Notified Basmati Varieties. Accessed: 20.04.2020. Available from: http://apeda.gov.in/apedawebsite/SubHead_Products/Basmati_Rice.htm.

25. Pomerantsev, A. L., \& Rodionova, O. Y. (2014). Concept and role of extreme objects in PCA/SIMCA. Journal of Chemometrics, 28(5), 429-438.

26. Worley, B., and Powers, R. (2013) Multivariate Analysis in Metabolomics. Current Metabolomics. 1(1), 92-107. 
27. Zontov, Y. V., Rodionova, O. Y., Kucheryavskiy, S. V., \& Pomerantsev, A. L. (2017). DD-SIMCA - A MATLAB GUI tool for data driven SIMCA approach. Chemometrics and Intelligent Laboratory Systems, 167, 23-28.

28. Broadhurst, D.I., and Kell, D.B. (2007). Statistical strategies for avoiding false discoveries in metabolomics and related experiments. Metabolomics, 2, 171-196.

29. Hastie, T., Tibshirani, R., and Friedman, J.H. (2009). The elements of statistical learning : data mining, inference, and prediction. Springer.

30. Westerhuis, J.A., Hoefsloot, H.C.J., Smit, S., Vis, D.J., Smilde, A.K., van Velzen, E.J.J., van Duijnhoven, J.P.M., and van Dorsten, F.A. (2008) Assessment of PLSDA cross validation. Metabolomics, 4, 81-89.

31. Xia, J., Mandal, R., Sinelnikov, I. V., Broadhurst, D., and Wishart, D.S. (2012). MetaboAnalyst 2.0--a comprehensive server for metabolomic data analysis. Nucleic Acids Research, 40, W127-W133.

32. Koo, I., Kim, S., \& Zhang, X. (2013). Comparative analysis of mass spectral matching-based compound identification in gas chromatography-mass spectrometry. Journal of Chromatography A, 1298, 132-138.

33. Kopka, J., Schauer, N., Krueger, S., Birkemeyer, C., Usadel, B., Bergmuller, E., Dormann, P., Weckwerth, W., Gibon, Y., Stitt, M., Willmitzer, L., Fernie, A. R., \& Steinhauser, D. (2005). GMD@CSB.DB: the Golm Metabolome Database. Bioinformatics, 21(8), 1635-1638.

34. Singh, V., Singh, A.K., Mohapatra, T., Krishnan S, G., and Ellur, R.K. (2018) Pusa Basmati 1121 - a rice variety with exceptional kernel elongation and volume expansion after cooking. Rice $11,19$. 
Table 1: Best performing multiclass models generated in SIMCA 15 and 'The Lab' based on all 7 varieties, Pusa 1121 and its potential adulterants and Taraori and its adulterants.

\begin{tabular}{|c|c|c|c|c|c|}
\hline \multicolumn{6}{|c|}{ Multiclass Models (SIMCA 15) } \\
\hline Model & M1 & M3 & M10 & M13 & M15 \\
\hline Model Type & PLS-DA & PLS-DA & OPLS-DA & PLS-DA & PLS-DA \\
\hline Pre-processing & Unprocessed & SNV & SNV & Unprocessed & SNV \\
\hline \% correct Pusa 1121 & $97.6 \%$ & $97.6 \%$ & $100.0 \%$ & - & - \\
\hline \% correct Pusa 1509 & $83.7 \%$ & $83.7 \%$ & $91.8 \%$ & - & - \\
\hline \% correct Sugandha & $92.8 \%$ & $92.8 \%$ & $96.4 \%$ & - & - \\
\hline$\%$ correct Taraori & $96.0 \%$ & $96.0 \%$ & - & $100.0 \%$ & $100.0 \%$ \\
\hline \% correct DB* & $92.5 \%$ & $92.5 \%$ & - & $98.5 \%$ & $98.5 \%$ \\
\hline$\%$ correct Shabnam & $100.0 \%$ & $100.0 \%$ & - & $98.0 \%$ & $98.0 \%$ \\
\hline$\%$ correct Sharbati & $98.0 \%$ & $98.0 \%$ & - & $100.0 \%$ & $100.0 \%$ \\
\hline Cut off & 0.36 & 0.36 & 0.44 & 0.44 & 0.45 \\
\hline & \multicolumn{5}{|c|}{ Multiclass models ('The Lab') } \\
\hline Model & RF18 & RF52 & RF54 & RF79 & \\
\hline Model Type & RF & RF & $\mathrm{RF}$ & $\mathrm{RF}$ & \\
\hline Pre-processing & $\begin{array}{l}\text { SNV -Avg } \\
\text { Dtrend }\end{array}$ & $\begin{array}{l}\text { Log SNV -Avg } \\
\text { 2DER Zscore }\end{array}$ & $\begin{array}{c}\text { Log SNV -Min 2DER } \\
\text { Zscore }\end{array}$ & $\begin{array}{l}\text { Log SNV } \\
\text { Zscore }\end{array}$ & \\
\hline \% correct Pusa 1121 & $97.6 \%$ & $100.0 \%$ & $100.0 \%$ & - & \\
\hline \% correct Pusa 1509 & $59.2 \%$ & $91.8 \%$ & $91.8 \%$ & - & \\
\hline \% correct Sugandha & $76.2 \%$ & $95.2 \%$ & $96.4 \%$ & - & \\
\hline$\%$ correct Taraori & $96.0 \%$ & - & - & $98.0 \%$ & \\
\hline \% correct DB* & $80.6 \%$ & $-\quad>P$ & - & $89.6 \%$ & \\
\hline$\%$ correct Shabnam & $96.4 \%$ & - & - & $97.6 \%$ & \\
\hline$\%$ correct Sharbati & $82.0 \%$ & - & - & $88.0 \%$ & \\
\hline F1 & 0.88 & 0.87 & 0.87 & 0.93 & \\
\hline
\end{tabular}


Table 2: Best performing binary models generated in SIMCA 15 and 'The Lab' based on Pusa 1121 vs its potential adulterants and Taraori vs its potential adulterants.

\begin{tabular}{lcccc}
\hline \multicolumn{5}{c}{ Binary models (SIMCA 15) } \\
\hline Model & M9 & M10 & M13 & M15 \\
\hline Model Type & PLS-DA & OPLS-DA & PLS-DA & PLS-DA \\
\hline Pre-processing & SNV & SNV & Unprocessed & Unprocessed \\
\hline \% correct Pusa 1121 & $100 \%$ & $100 \%$ & - & - \\
\hline \% correct Taraori & - & - & $100 \%$ & $100.0 \%$ \\
\hline \% correct Adulterant & $100 \%$ & $100 \%$ & $100 \%$ & $100 \%$ \\
\hline Cut off & $\mathbf{0 . 7 3}$ & $\mathbf{0 . 7 3}$ & $\mathbf{0 . 5 5}$ & $\mathbf{0 . 5 1}$ \\
\hline \multicolumn{7}{c}{ Binary Models ('The Lab') } & & \\
\hline Model & RF109 & RF110 & RF138 & RF139 \\
\hline Model Type & RF & RF & RF & RF \\
\hline Pre-processing & Log SNV - & Log SNV - & Log SNV - & Log SNV - \\
& Avg 1DER & Avg 2DER & Avg 1DER & Avg 2DER \\
\hline \% correct Pusa 1121 & Zscore & Zscore & Zscore & Zscore \\
\hline \% correct Taraori & $100 \%$ & $100 \%$ & - & - \\
\hline \% correct Adulterant & - & - & $100 \%$ & $100.0 \%$ \\
\hline F1 & 98.5\% & $98.5 \%$ & $98.5 \%$ & $98.5 \%$ \\
\hline
\end{tabular}




\section{Journal Pre-proof}

Figure 1: A) Raw NIR spectra of all rice varieties in the 'The Lab' software B) Multiclass OPLSDA model (SNV, 1stDer and SG) containing all 7 Indian basmati rice varieties.
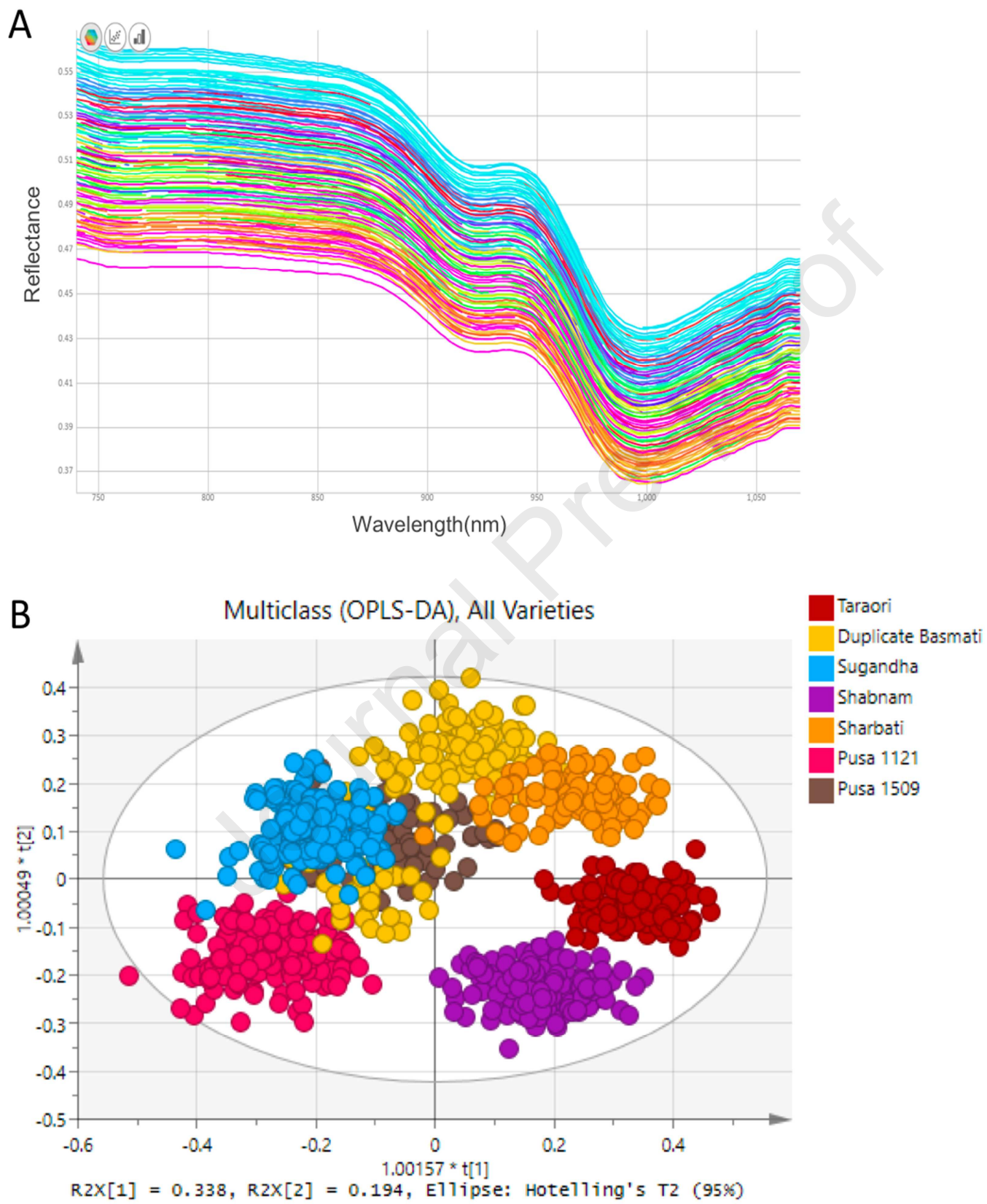
Figure 2: Chemometric models to class various Basmati varieties of rice A) Binary OPLS-DA model (SNV, 1stDer and SG) containing Pusa 1121 and its potential adulterants and, b) Binary OPLS-DA model (SNV, 1stDer and SG) containing Taraori and its potential adulterants.
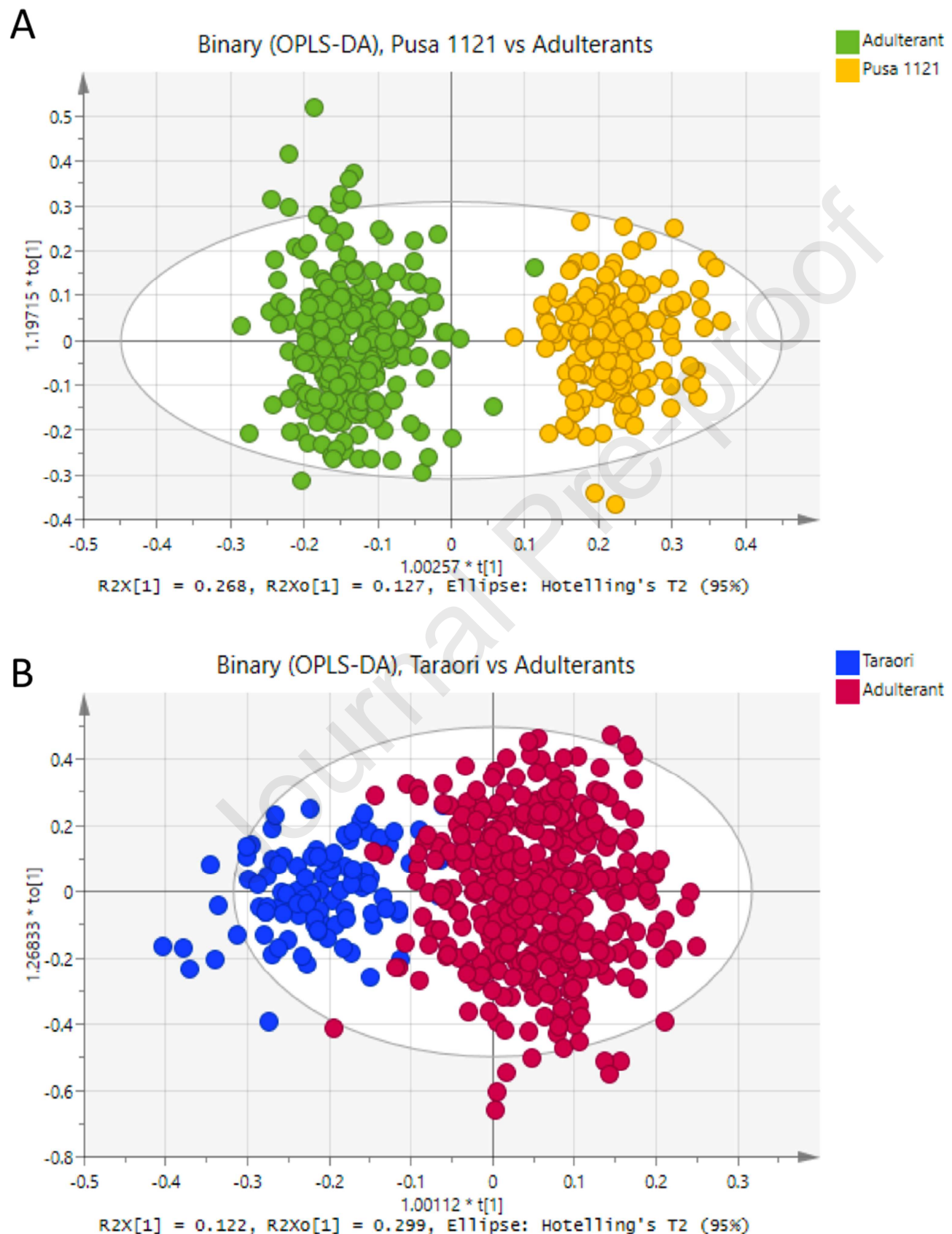
Figure 3: The application of DD-SIMCA for the classification of high quality 1121 and Taraori rice samples from adulterants. A) 1121 ( $n=167)$ vs 1509 and Sugandha $(n=132)$; B) Taraori rice samples $(n=100)$ vs $D B$, Sharbati and Shabnam rice samples $(n=200)$; Corresponding extreme plots for each training set shown next to the acceptance plots. The acceptance plot for training set provides a graphic representation of the acceptance area, the area inside the green curve with the threshold for $\alpha=0.01$. Authentic samples falling outside the green curve were considered extremes represented with orange box. C) Summarization of the performance of the DD-SIMCA models for rice samples 1121 vs 1509, Sugandha rice samples and Taraori vs DB, Sharbati, Shabnam rice samples.

A

1121

vs 1509 \& Sugandha

Extreme plot
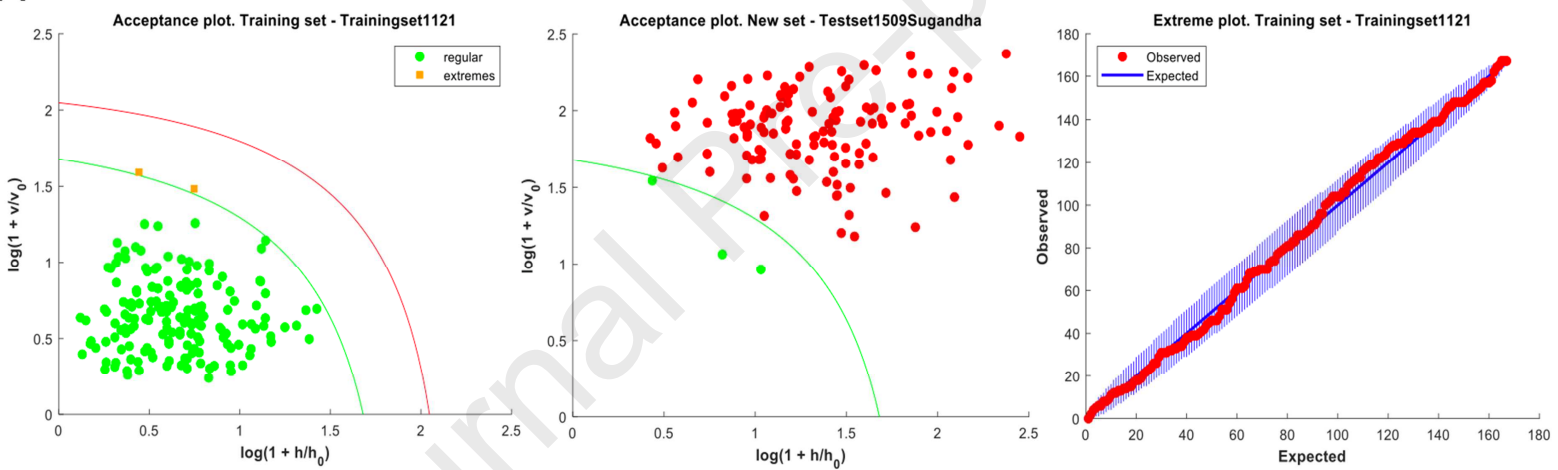

B

vs DB, Sharbati \& Shabnam

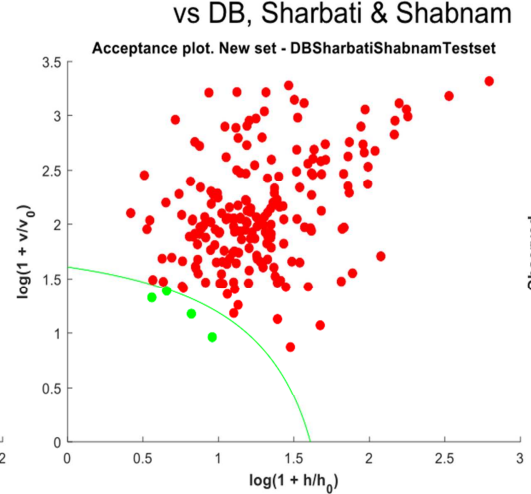

Extreme plot
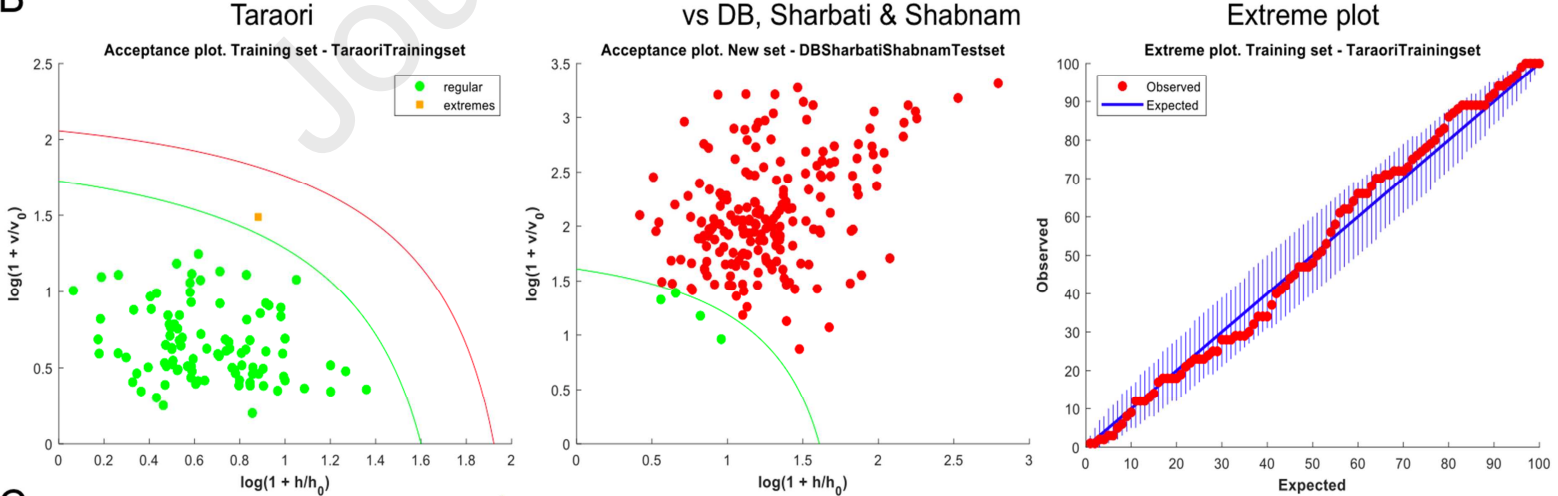

C

\begin{tabular}{|l|l|l|l|l|l|l|l|}
\hline Target & PCs & $\boldsymbol{\alpha}$ & $\mathbf{V}$ & DOF (SD) & DOF (OD) & SEN & SPE \\
\hline 1121 vs 1509 \& Sugandha & 5 & 0.01 & 0.01 & 6 & 6 & $98.8 \%$ & $97.7 \%$ \\
\hline Taraori vs DB, Sharbati \& Shabnam & 5 & 0.01 & 0.01 & 7 & 6 & $98 \%$ & $99 \%$ \\
\hline
\end{tabular}


Figure 4: The application of DD-SIMCA for the classification of high quality 1121 and Taraori rice samples from individual adulterants. A) 1121 ( $n=167)$ vs 1509 ( $n=49)$, and Sugandha $(n=83) ;$ B) Taraori rice samples $(n=100)$ vs DB $(n=67)$, Sharbati $(n=50)$ and Shabnam $(n=82)$ rice samples; C) Summarization of the performance of the DD-SIMCA models for all rice samples.
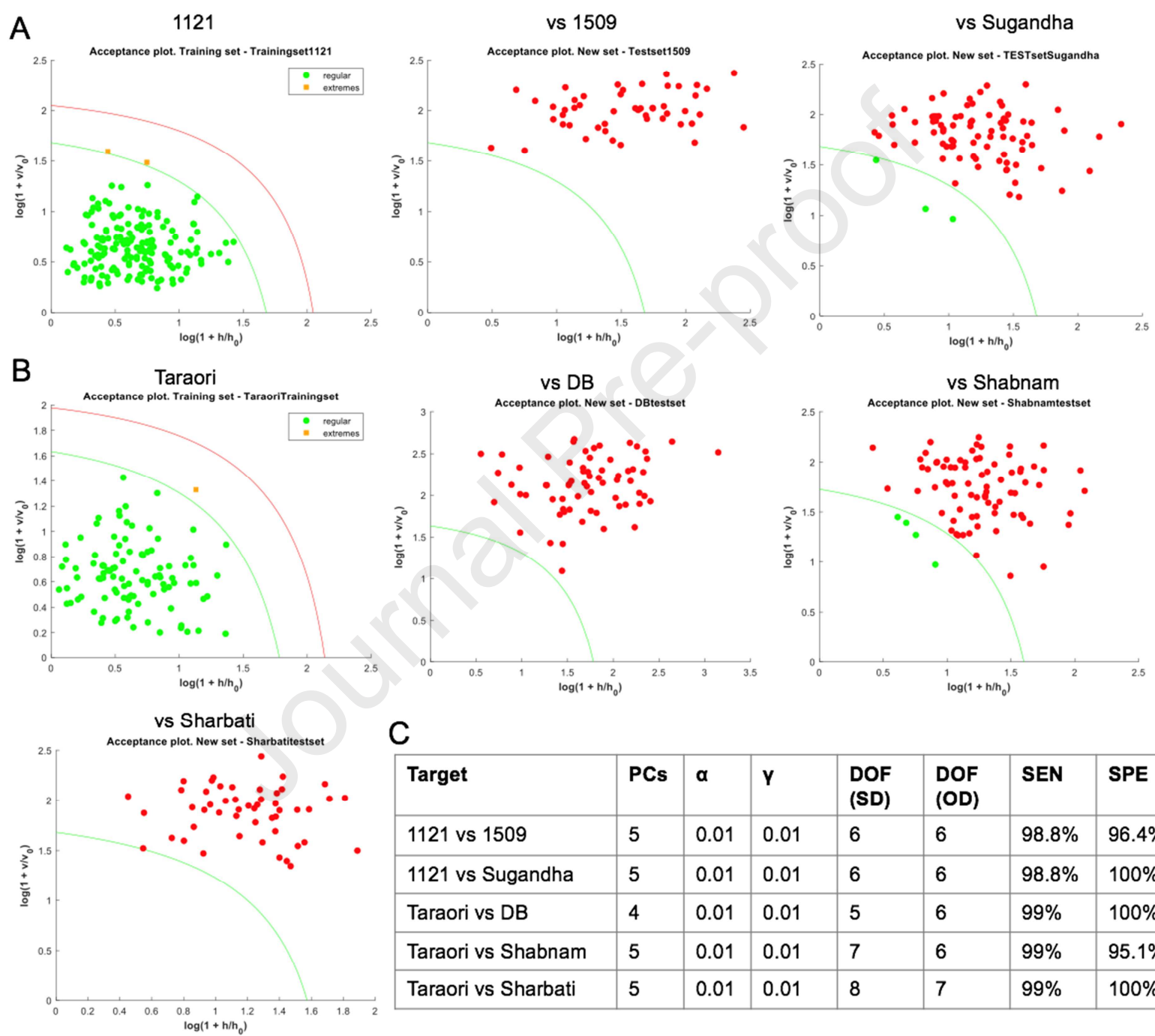

C

\begin{tabular}{|l|l|l|l|l|l|l|l|}
\hline Target & PCs & $\boldsymbol{\alpha}$ & $\mathbf{Y}$ & $\begin{array}{l}\text { DOF } \\
\text { (SD) }\end{array}$ & $\begin{array}{l}\text { DOF } \\
\text { (OD) }\end{array}$ & SEN & SPE \\
\hline 1121 vs 1509 & 5 & 0.01 & 0.01 & 6 & 6 & $98.8 \%$ & $96.4 \%$ \\
\hline 1121 vs Sugandha & 5 & 0.01 & 0.01 & 6 & 6 & $98.8 \%$ & $100 \%$ \\
\hline Taraori vs DB & 4 & 0.01 & 0.01 & 5 & 6 & $99 \%$ & $100 \%$ \\
\hline Taraori vs Shabnam & 5 & 0.01 & 0.01 & 7 & 6 & $99 \%$ & $95.1 \%$ \\
\hline Taraori vs Sharbati & 5 & 0.01 & 0.01 & 8 & 7 & $99 \%$ & $100 \%$ \\
\hline
\end{tabular}


Figure 5: A) PCA scores plot for the first component (53.6\%) Vs second component (22\%), indicating clustering of sample groups, B) Graphical representation of differential levels of VOCSs of Pusa 1121, Pusa 1509 and Sugandha sample groups. C) a) PCA scores plot for the first component (54.4\%) Vs second component (16.9\%), indicating clustering of sample groups, b) Graphical representation of differential levels of VOCSs of Duplicate Basmati, Shabnam, Sharbati and Taraori sample groups.

A

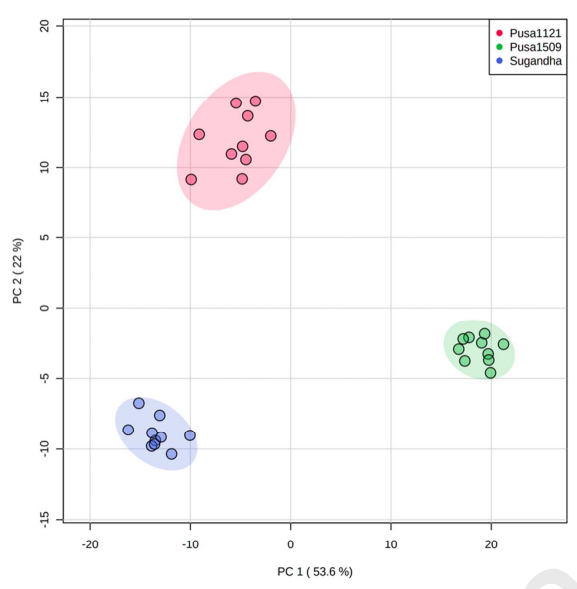

C

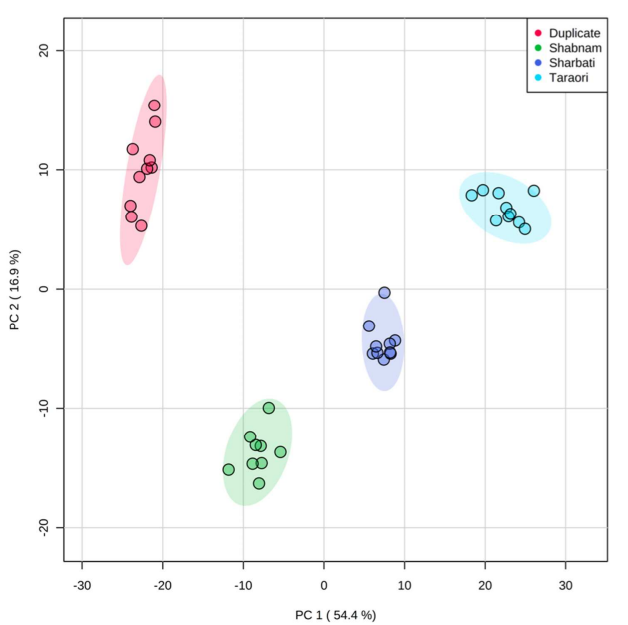

B

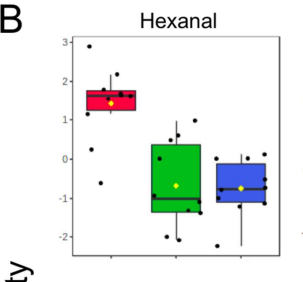

Heptanoic acid

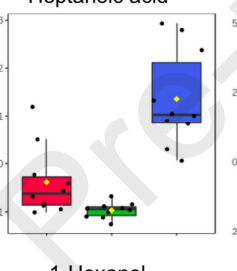

1-Hexano

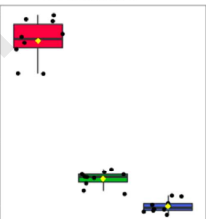

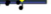

Sample groups

D
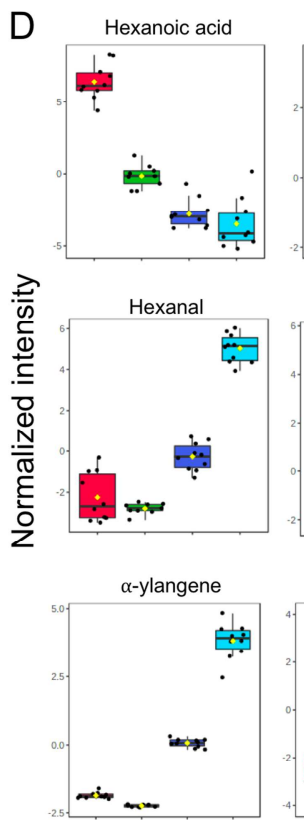

Nonanal

Nonanoic acid

3-Nonene-1-ol

1-hexanol-2-ethyl

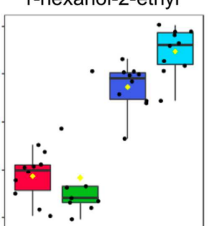

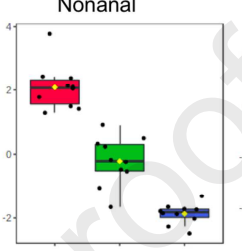

Heptanal
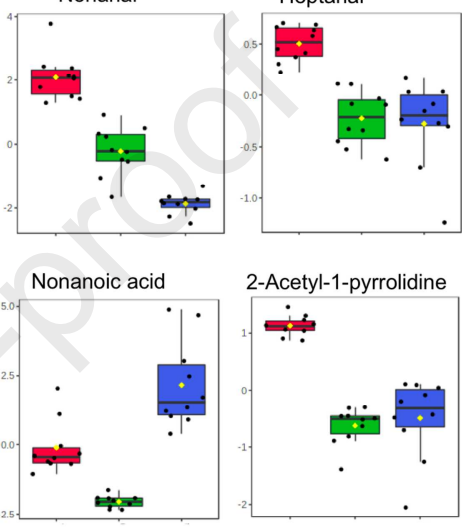

2-Acetyl-1-pyrrolidine

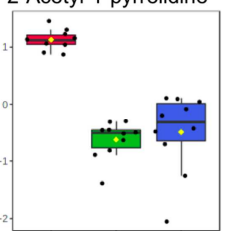

Hexane,2,3,4-trimethyl
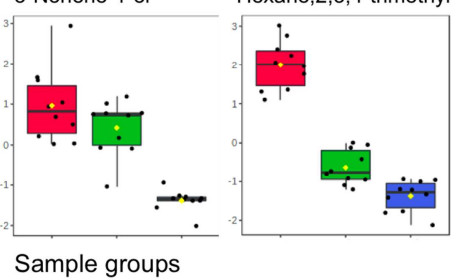

$\because \because$
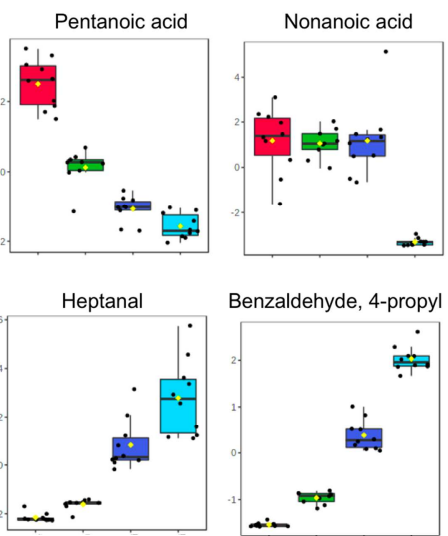

Benzaldehyde, 4-propyl

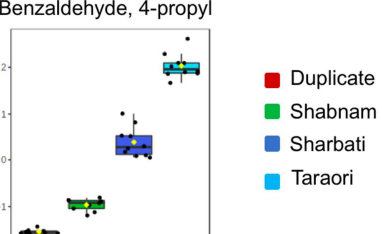

Propanal-2methyl

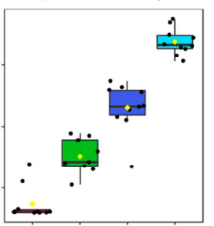

Sample groups 
1. Basmati rice fraud is a major problem worldwide.

2. A two-tiered testing system can help address this fraud issue.

3. Rapid on-site screening of samples using handheld portable NIR.

4. HS-GC-MS is then used for confirmatory analysis of suspect samples.

5. Chemometrics successfully separates authentic rice from adulterants. 